\title{
THE POLYADIC COMPLETION \\ OF A TRANSFORMATION ALGEBRA
}

\author{
ISIDORE FLEISCHER
}

(Communicated by Andreas R. Blass)

\begin{abstract}
The (faithful) polyadic completion was constructed in a special case using $\{0,1\}$-valued homomorphisms by Leblanc. Here a different methodadjoining extrema freely to the underlying Boolean algebra-succeeds in full generality. This is based on an apparently new axiomatization of locally finite polyadic algebras of infinite degree within the class of transformation algebras as those having certain extrema that the transformations preserve.
\end{abstract}

\section{INTRODUCTION}

An algebraic description of the predicate calculus with equality was developed around 1950 by Tarski and his collaborators. These "cylindric algebras" are Boolean algebras with unary operators to represent the existential quantifications and a doubly indexed family of Boolean elements which function as those formulae that equate two variables. Replacement of one variable by another in a formula, say $x$ by $y$ in $\varphi$, is defined to be (the algebraic counterpart of) $\exists x(x=y \wedge \varphi)$. Subsequently, Halmos treated the predicate calculus without equality algebraically by dropping the equality elements and incorporating the single variable replacements as a doubly indexed family of primitive unary operators to complement the quantificational operators. Halmos actually introduced unary operators for simultaneous (multiple) variable replacements but pointed out $[\mathrm{H}, \mathrm{p} .146]$ that for finitary formulae when there are infinitely many variables, the single replacements suffice. In the course of his expository description of these "polyadic algebras" Halmos briefly introduced "transformation algebras", which are Boolean algebras equipped with only the transformational portion of a polyadic structure. These algebras were taken up by Leblanc who set out to base algebraic logic on the transformation structure alone, insofar as possible. It is known that in the Lindenbaum algebra of a predicate theory, the quantifications of a variable are extrema for every infinite set of its replacement instances: Leblanc carries this through in the polyadic setting [L, 1.3 Lemma] thus essentially effecting a reduction of the polyadic to the transformation (plus order) structure. Leblanc goes on to characterize those transformation algebras that underlie polyadic algebras [L, p. 605]. A different characterization is given

Received by the editors November 26, 1990.

1991 Mathematics Subject Classification. Primary 03G05, 03G15, 03B10, 03 B30. 
below-the subsets of single variable replacement by infinitely many variables must have extrema that are preserved by replacement-and this leads to a new description of the polyadic completion and a proof of its faithfulness, valid in general.

\section{Preliminaries}

As originally defined, a "transformation algebra" is a Boolean algebra $B$ equipped with an endomorphic monoid action by the self-maps of an auxiliary set $X$ of "variables": what this comes to is an assignment, to every self-map $\tau$ of $X$, of a Boolean endomorphism on $B$ (which it will not harm to denote with the same symbol) such that the identity self-map on $X$ is assigned the identity endomorphism on $B$ and the composite on $X$ is assigned the composite on $B$. Variable dependence can be defined with the help of the self-maps that leave all but one variable fixed: the "replacement of $x$ by $y$ ", written $(x \mid y)$, sends $x$ to $y$ and fixes all $z \neq x$. A $\varphi \in B$ is "independent of" $x$ if some replacement of $x$ fixes it (then all replacements do, since they absorb each other); $x$ "occurs in" $\varphi$ if some (hence all) replacements of $x$ move it. For the usual formal languages, the set $X$ of variables is infinite while every element of $B$ absorbs every transformation which fixes some finite set of variables. In the presence of this "local finiteness" a couple of simplifications can be made in the definition of transformation algebra. Since the image of $\varphi$ by any transformation is the same as by that which agrees with it on this finite set and fixes the complement, the full transformational action is determined by that of the submonoid of self-maps which fix all but finitely many variables; moreover, the action of each of these on a $\varphi$ coincides with a finite composite of replacements. A transformation algebra may thus be defined as a Boolean algebra equipped with an endomorphic action by replacements such that composites of these that agree as transformations on $X$ also agree as endomorphisms on $B$.

A set $\{(x \mid y) \varphi\}$ ( $x$ and $\varphi$ fixed, $y$ varying) is called an "existential" subset (it includes $\varphi$ since $(x \mid x)$ is the identity); its supremum over $y$, if it exists, is denoted $\exists x \varphi$. The variable of quantification $x$ is "dummy": it may be replaced by any other $z$ not occurring otherwise in $\varphi$, since $(z \mid y)(x \mid z) \varphi=$ $(x \mid y)(z \mid x) \varphi=(x \mid y) \varphi$ entails $\exists z(x \mid z) \varphi=\exists x \varphi$. This shows that $\exists x \varphi$ is the sup of $(x \mid y) \varphi$ for any infinite set of $y$ : for if $\psi$ dominates $(x \mid z) \varphi$ for a $z$ occurring in neither $\psi$ nor $\varphi$ then $\psi=\exists z \psi \geq \exists z(x \mid z) \varphi=\exists x \varphi$.

\section{Axiomatization}

A (locally finite) polyadic algebra may be defined as a transformation algebra in which all the suprema $\exists x \varphi$ exist and are preserved by the action of the replacements. In every transformation algebra, replacements preserve the sets of infinite replacement instances $(x \mid u)$ for a variable $x$ in a $\varphi:$ Indeed, $(x \mid y)$ is absorbed by the $(x \mid u)$ and $(z \mid y)$ commutes with the $(x \mid u)$ for $x \neq z, y$ except for $(x \mid z)$, which it converts to $(x \mid y)$. (To handle the action of $(z \mid x)$ change the dummy $x$ to a different variable not occurring in the argument $\varphi$.) The polyadic requirement is that $(x \mid y)$ send suprema on suprema.

In the usual polyadic axiomatization, the transformation formalism is augmented with quantifiers, introduced as additional primitive unary operators, while the postulates ensure that $\exists x$ absorbs $x$-replacement, is absorbed by it, 
and commutes with replacements of, and quantifiers in, the other variables. Conversely, this more elaborate axiomatization can be deduced from the above: since $\exists x$ and the $(x \mid y)$ absorb each other, the increasing isotone $\exists x$ is a closure operator to the common fixpoints of the $(x \mid y)$, which are a Boolean subalgebra; thus $\exists x$ is a polyadic quantifier. Its commuting with replacements of other variables entails that these quantifiers pairwise commute (since the iterated sup is the composite sup) whence their composite is their join, the quantifier to their common fixed points. To say that $x$-replacement is absorbed by $\exists x$ is to say that it acts on its image as the identity; more generally, every transformation has the same effect on $\exists x \varphi$ as does its modification that fixes $x$ (if the former sends $x$ to $y$ its effect is the same as the latter's preceded by a $(z \mid y)(x \mid z)$ absorbed by $\exists x \varphi)$, hence the same effect as any transformation differing only at $x$. Finally, if $\tau$ sends $x$ and nothing else on $y$, then it can be written $(x \mid y) \tau^{\prime}$ where $\tau^{\prime}$ fixes $x$ and does not map on $y$, hence $\tau \exists x=\exists x \tau^{\prime}=\exists y(x \mid y) \tau^{\prime}=\exists y \tau$; for several $x$ 's each the unique preimage of a corresponding $y \in Y$, this is $\tau \exists\left(\tau^{-1} Y\right)=\exists Y \tau$ (which may also be used to cover the absorbability of $\exists x$ by $(x \mid y)$ by admitting $y$ 's with no preimage and construing $\exists \varnothing$ to be the identity operator).

\section{THE POLYADIC COMPLETION}

Since every Boolean algebra is embeddable in a complete algebra (e.g., its MacNeille completion), it is embedded in the algebra freely generated over itself by suprema adjoined for a collection of its subsets. This extension $A$ of $B$ is characterized as admitting unique extension of morphisms from $B$ to any algebra in which the images of these subsets have suprema, so as to preserve them. $A$ may be obtained as a subdirect product over the algebras generated, over homomorphic images of $B$, by suprema for images of these subsets. It is worth remarking that an adjoined sup dominates an element $\varphi$ of $B$ only if some finite subsup does: for the elements dominated by these finite subsups constitute an ideal in $B$ which, if disjoint from $\varphi$, can be enlarged to a disjoint maximal ideal thus yielding a two-valued morphism that extends to $A$ to send $\varphi$ on 1 and the given sup on 0 . (More generally, $\varphi$ is dominated by a $\psi \in A$ if and only if it is when the adjoined sups used in generating $\psi$ are replaced by sufficiently large finite subsups.)

For $B$ a transformation algebra, adjoin freely suprema for the existential subsets $\{(x \mid y) \varphi\}$ for each $x$ and $\varphi$. It will first be shown that each such sup in $A$ is equally the sup of any subset obtained by supressing finitely many of the $(x \mid y) \varphi$. Since $A$ is generated over $B$ by the existential sups, its elements are finite infs of finite sups of these generators and their complements; whence it suffices to show that a finite sup of these, which dominates $(x \mid y) \varphi$ for cofinitely many $y$, dominates all. If the finite sup combines any complements of existential sups, i.e., "universal infs", it suffices to replace these by their terms, for if each of these finite sups of terms dominates an element, so will the original finite sup by infinite distributivity. There remains a finite sup of adjoined sups and elements of $B$, which may be construed as a single infinite sup $\psi$, to which the argument in the preceding paragraph applies. So let a finite subsup of $\psi$ dominate $(x \mid z) \varphi$ for a $z$ occurring neither in $\varphi$ nor fixed in this bounding sup $\psi$ : then the replacements for $z$ in $(x \mid z) \varphi$ will yield all the $(x \mid y) \varphi=(z \mid y)(x \mid z) \varphi$, 
each of which will be dominated by some finite subsup of $\psi$, hence by $\psi$.

That the adjoined sups are unchanged under suppression of finitely many terms permits extending the Boolean endomorphisms from $B$ to $A$ : for $(x \mid y)$ is absorbed by the $(x \mid u)$ and commutes with $(z \mid u)$ for $z \neq x, y$ except for $(z \mid x)$ which it converts to $(z \mid y)$. By the universal property of $A$, the $(x \mid y)$ extend to endomorphisms of $A$ so as to be absorbed by the adjoined $\bigvee(x \mid u)$ and to commute with the adjoined $\bigvee(z \mid u)$ for $z \neq x, y$ (use that $(y \mid u) \varphi=(z \mid u)(y \mid z) \varphi$ for $z$ not occurring in $\varphi$ to see that $(x \mid y)$ preserves the remaining subsets for which sups have been adjoined). By the uniqueness of the extension it preserves equalities between composites of the $(x \mid y)$ hence equips $A$ with a transformation structure; this is moreover locally finite since $\bigvee(x \mid u) \varphi$ is independent of the same variables as $\varphi$ (as well as of $x$ ).

Thus the construction can be repeated with $A$ in place of $B$, the suprema in $A$ of existential subsets in $B$ being retained by universality. Iterating, one obtains an increasing sequence of transformation algebras such that every existential subset in one has a supremum in the next, retained in all subsequent ones. The union of this chain is a transformation algebra with suprema for each of its existential subsets which the replacements preserve, hence, by the axiomatization presented above, is a polyadic (thus the free) completion.

\section{REFERENCES}

[H] P. R. Halmos, Algebraic logic, Chelsea, New York, 1962.

[HMT] L. Henkin, D. Monk, and A. Tarski, Cylindric algebras, Part I, North-Holland, Amsterdam, 1971; Part II, 1985.

[L] L. Leblanc, Transformation algebras, Canad. J. Math. 13 (1961), 602-613.

Department of Mathematics, University of Windsor, Windsor, Ontario, Canada N9B 3P4 